\title{
Emerging Next Generation Solar Cells: Route to High Efficiency and Low Cost
}

\author{
M. S. Sadek ${ }^{*}$ \\ Dept. of Electrical and Electronic Engineering, \\ Southeast University, \\ Dhaka, Bangladesh \\ M.A. Zaman \\ Dept. of Electrical and Electronic Engineering, Prime \\ University \\ Dhaka, Bangladesh
}

\begin{abstract}
Generation of clean energy is one of the main challenges of the 21 st century. Solar energy is the most abundantly available renewable energy source which would be supplying more than $50 \%$ of the global electricity demand in 2100. Solar cells are used to convert light energy into electrical energy directly with an appeal that it does not generate any harmful bi-products, like greenhouse gasses. The manufacturing of solar cells is actually based on the types of semiconducting or non-semiconducting materials used and commercial maturity. From the very beginning of the terrestrial use of Solar Cells, efficiency and costs are the main focusing areas of research. The definition of so-called emerging technologies sometimes described as including any technology capable of overcoming the ShockleyQueisser limit of power conversion efficiency (33.7 percent) for a single junction device. In this paper, few promising materials for solar cells are discussed
\end{abstract}

\author{
M. J. Rashid \\ Dept. of Electrical and Electronic Engineering, \\ University of Dhaka, \\ Dhaka, Bangladesh \\ Z. H. Mahmood \\ Dept. of Electrical and Electronic Engineering, \\ University of Dhaka, \\ Dhaka, Bangladesh
}

including their structural morphology, electrical and optical properties. The excellent state of the art technology, advantages and potential research issues yet to be explored are also pointed out.

Keywords -Solar Cells, Thin film, Silicon, Organic, Perovskite, DSSC, CZTS, Efficiency, Costs.

\section{INTRODUCTION}

Based on different technologies and materials, the solar cells can be grouped into three different generations. First generation or c-Si solar cells are most dominant photovoltaic (PV) technology even today for their good performance and better stability; typically demonstrate efficiency about 22\%. [1] However, rigidity, complex manufacturing processes and high costs still remains as bottle neck. The second generation or thin film solar cells demonstrate typical efficiency in $10-15 \%$.[3]Although the efficiency is 
less than the c-Si solar cells, the comparative low cost makes it an attractive alternative.

The third generation solar cells employ the most advanced techniques which is basically thin film based too, but the varieties of materials used to manufacture such cells introduces a new era of cell design. These cells are made of semiconducting p-n junctions ("first generation") and thin film cells ("second generation"). Common third-generation system techniques include multi-layer ("tandem") cells made of amorphous silicon or III-V compounds like gallium arsenide, frequency up down conversion, Intermediate band, hot-carrier effects and other multiple-carrier ejection techniques.[1-4]

In the most advanced PV technology, a wide range of materials, ranging from semiconducting, nonsemiconducting, different polymers and even organic are incorporated which actually broadens the choice of materials to manufacture solar cell devices. Not only materials aspect, but also the uses of cutting edge technologies like tandem design of cells, Intermediate band concept, Hot carrier cell, Hybridization of both inorganic and organic materials shows a lots of promises in designing such cells.Some examples of such cell design include - Perovskite solar cell, Copper Zinc Tin Sulfide solar cell (CZTS), and derivate CZTSe and CZTSSe. Dye-Sensitized Solar Cell(DSSC), also known as "Grätzel cell"

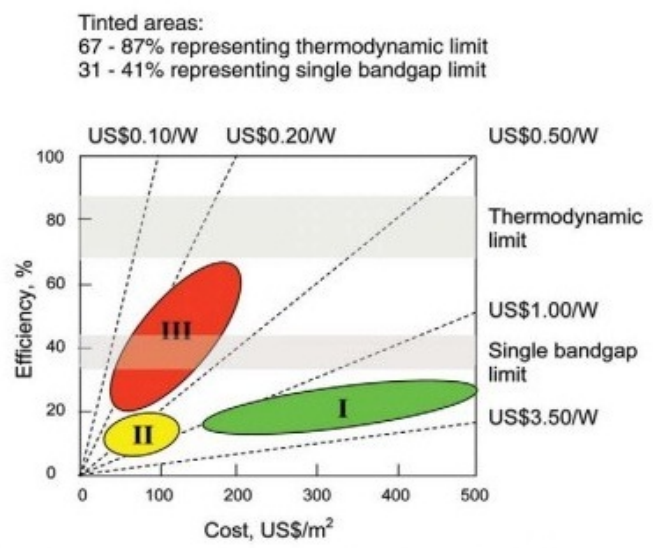

Fig.1.Graph of efficiency vs. cost for generations of solar cells. [M. A. Green, "Third Generation Photovoltaics", Springer Verlag, 2003]

\section{Perovskite Solar Cell}

A perovskite solar cell is a type of solar cell which includes a perovskite structured compound, most commonly a hybrid organic-inorganic lead or tin halide-based material, as the light-harvesting active layer. [5]

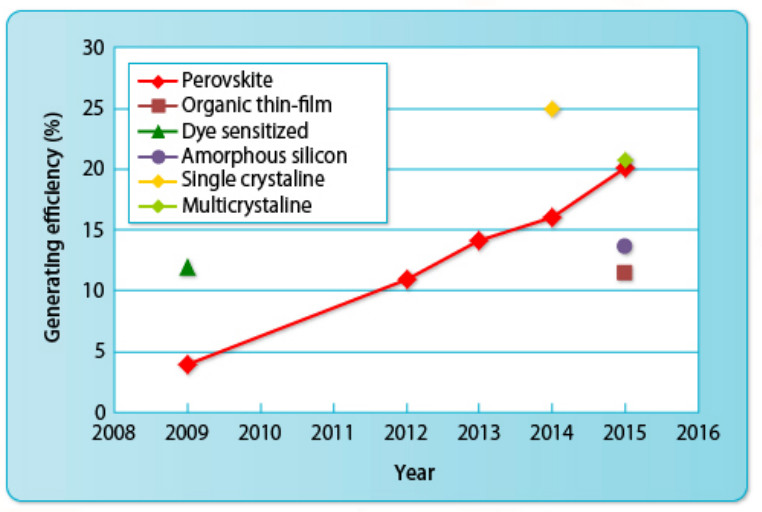

Fig. 2. Trends in maximum conversion efficiency for solar cells at the research stage Source: Created based on the NREL homepage http://www.nrel.gov/ncpv/images/efficiency_chart.jpg 
The efficiency has significantly rises from $3 / 8 \%$ in conduction band of $\mathrm{TiO}_{2}$ and collected at the $2009[6]$ to $21.0 \%$ in 2015[7] by using methylammonium lead halides $\left(\mathrm{CH}_{3} \mathrm{NH}_{3} \mathrm{PbX}_{3}\right)$ materials as absorber layer which are cheap and simple to manufacture. With the potential of achieving even higher efficiencies and the very low production costs, perovskite solar cells have become commercially attractive, with start-up companies already promising modules on the market by 2017. [8,9]

Traditional silicon cells require expensive, multistep processes, conducted at high temperatures $\left(>1000^{\circ} \mathrm{C}\right)$ in a high vacuum in special clean room facilities. [10] Meanwhile the organic-inorganic perovskite material can be manufactured with simpler wet chemistry techniques in a traditional lab environment.

A. Perovskite Solar Cell Materials, Structure and operating principles

The most commonly studied perovskite absorber is methylammonium lead trihalide $\left(\mathrm{CH}_{3} \mathrm{NH}_{3} \mathrm{PbX}_{3}\right.$, where $\mathrm{X}$ is a halogen atom such as iodine, bromine or chlorine), with an optical bandgap between $1.5 \mathrm{eV}$ and $2.3 \mathrm{eV}$ depending on halide content. So by introducing bandgap tailoring, the optical properties of this material can be optimized.

Incoming radiation from the sun is absorbed by the Halide perovskite which is integrated with mesoporous $\mathrm{TiO}_{2}$ thin film. Electrons generated due to light absorption gets excited and move to the conduction band of perovskite and then jumps to the electrode. These missing electrons are replenished by a HTM (Hole Transport Material) mechanism.
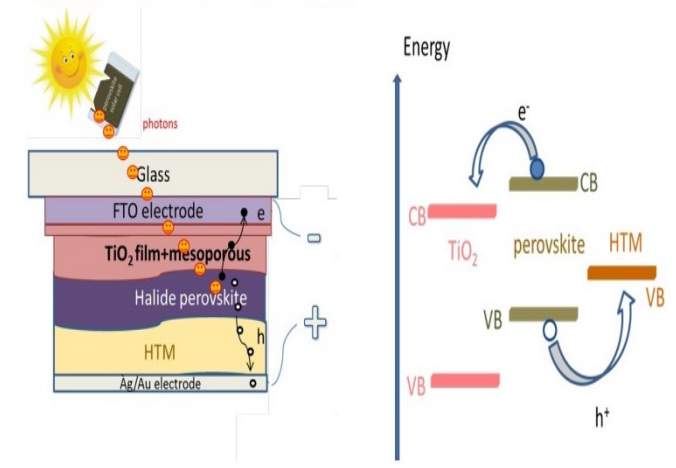

Fig.

3.Perovskite Solar Cell structure and basic working procedure

There are some very prominent features which makes perovskite solar cell as one of the best emerging PV technologies, which includes-

- Simple manufacturing process (as compared to the dominant Si based PV technology). [11]

- Bandgap modification by changing the Halide components as well as their proportion. [12]

- Perovskite materials have very low recombination rate. So display a long diffusion length for both holes and electrons of over one micron [13, 14]. The long diffusion length means that these materials can function effectively in thin-film architecture, and that charges can be transported in the perovskite itself over long distances.

- It has recently been reported that charges in the 
perovskite material are predominantly present as free electrons and holes, rather than as bound excitons, since the exciton binding energy is low enough to enable charge separation at room temperature.[15]

Although perovskite Solar Cells exhibiting higher conversion efficiency with relatively low costs, but there are some areas which need to bemastered -

The prime challenges for perovskite solar cells are inclusion of lead which is detrimental to the device performance and the aspect of short-term and longterm stability. The water-solubility of the organic constituent of the absorber material makes devices highly prone to rapid degradation in moist environments. [16] However, no long term studies and comprehensive encapsulation techniques have yet been demonstrated for perovskite solar cells.

Beside moisture instability, it has some other constructional issues need to be addressed. This includes -

- Protective Glass - transparent but not flexible, so it can't be applicable to BIPV type applications.

- ITO/FTO electrode - In and Sn are deficient materials. In is basically used for display purpose and $\mathrm{F}$ is harmful for environment and human health. may exist better electrons transporter materials.

- Holes transporter (HTM): It is not stable in ambient atmosphere

\section{B. Perovskite-Si Tandem Cell Design}

Perovskite cells showing record efficiency beyond $20 \%$, not only efficient but also have a high and tunable bandgap, cheap fabricating techniques makes it suitable for application in tandem devices.
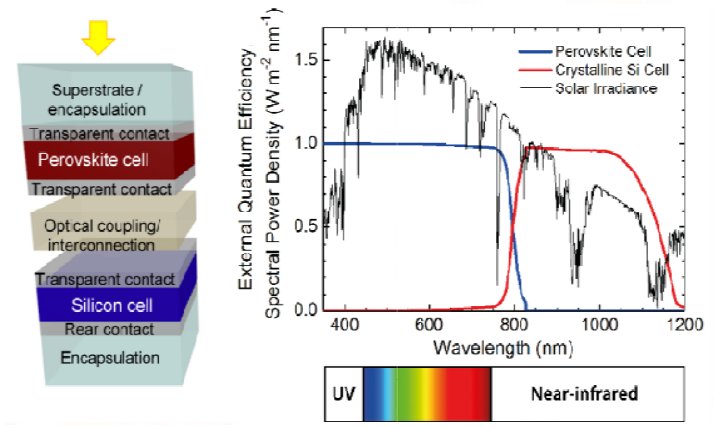

Fig.4. Perovskite-Si tandem structure and spectral response

As this silicon cell efficiency record is already very close to the practical efficiency limit of this type of solar cells, perovskite/silicon tandem cells with their potential for ultra-high efficiencies at affordable costs represent the most straight forward way to decrease the overall cost of photovoltaic systems and consequently also to reduce the price of electricity for end-users.

- TiO2 layers - Acts as electron transporter but, 


\section{CZTS (Copper Zinc Tin Sulfide) Solar Cell:}

Copper-zinc-tin-sulfur CZTS-based technology uses materials that avoid heavy metals, earth abundant and also at a lower cost. The constituents of this semiconductor material copper $(\mathrm{Cu}), \operatorname{zinc}(\mathrm{Zn}), \operatorname{tin}(\mathrm{Sn})$ and sulfur(S), have the advantages of being both abundant in the earth's crust and non-toxic.

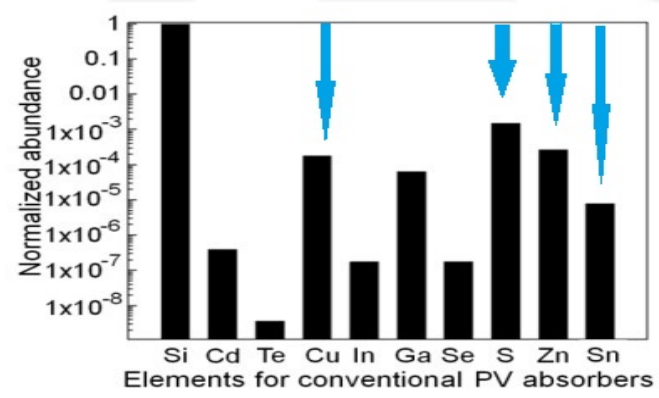

Fig. 5. Normalized abundances of some common PV absorber materials

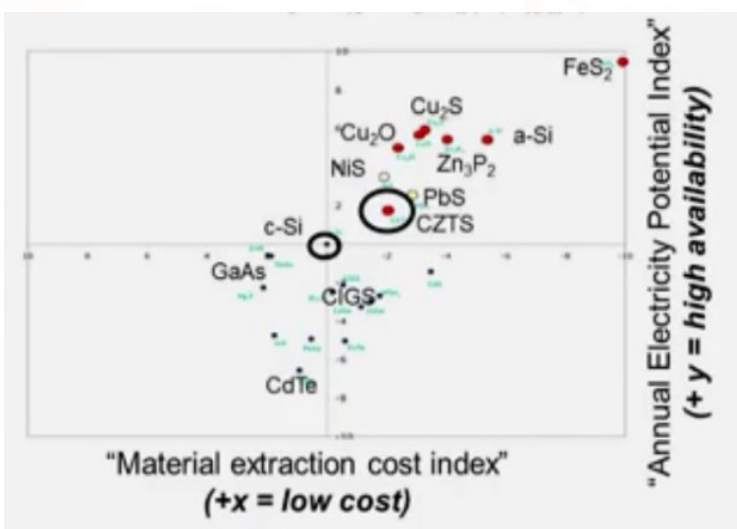

Fig. 6.Cost Vs Availability curve of the most used solar cell manufacturing materials along with CZTS.

CZTS also has near ideal properties for solar photo voltaics, as it is a very strong absorber and has a band gap of around $1.4 \mathrm{eV}$. IBM has an active research department in this area and recently announced a CZTS based solar cell that had a conversion efficiency of $11.1 \%$ and could potentially be printed. [17] This efficiency is lower than the equivalent laboratory records of $19.6 \%$ for CIGS and $17.3 \%$ for CdTe, however CZTS is at a much earlier stage of development. [18]

Some key factors make the IBM solar cells become a viable alternative to existing "thin film" solar cells. These includes -

Firstly - It uses rather earth abundant, Nontoxic elements as the constituents. It is well known that in case of CdTe based cell, Tellurium are extremely rare, and $\mathrm{Cd}$ is toxic. And in case of CIGS, Indium and Gallium are rare and most widely used in displays.

Secondly- An inexpensive ink-based manufacturing process. According to a study done by IBM, thin film solar cells will likely be limited to producing about 0.3 terawatts, but the new cells from IBM could produce an order of magnitude more power. [19]

CZTS has numerous advantages that could lead to its massive use as an abundant, non-toxic, low cost absorber for thin film photovoltaic solar cells -

- it is a compound whose intrinsic point defects lead to p-type semiconductor behavior. Although such intrinsic point defects levels lead to low charge separation, which can be overcome by thanks to the type-II band alignment of the $\mathrm{CZTS} / \mathrm{CdS}$ 
interface. Incorporation of $\mathrm{CdS}$ buffer layer provide excellent band offset and also improves the efficiency a bit. [20]

- it has a direct bandgap and an absorption coefficient $>10^{4} \mathrm{~cm}^{-1}$, which is suitable for thin film photovoltaic applications [21-23]

- Band gap ofthe CZTS thin film can modified from $0.94 \mathrm{eV}$ to $1.6 \mathrm{eV}$ by changing the zinc and tin composition and replacing the selenium $(\mathrm{Se})$ by sulfur (S). CZTS belongs to the Kesterites structure and composed of $\mathrm{Cu} 2 \mathrm{ZnSnS} 4$ (CZTS), $\mathrm{Cu} 2 \mathrm{ZnSnSe} 4$ (CZTSe) and CZTSSe are the competitors to the CIGS and CdTe based thin film solar cells. This tenability is of particular interest for the manufacturing of absorbers with a band gap between 1.1 and $1.5 \mathrm{eV}$, which allow theoretical efficiencies higher than $30 \%$ [24]

- It includes $\mathrm{Zn}$ and $\mathrm{Sn}$ which are respectively produced in quantity 20000 and 500 times bigger than In which is one of the constituents materials of CIGS and moreover Indium is used for display devices. [25]

- As CZTS solar cells uses the same structural morphology as CIGS, so the knowledge gathered on back contact, buffer layers and window layer by CIGS scientists can therefore be used and adapted for CZTS solar cells.
A. Potential research issues for CZTS Solar Cells

There are still numerous hurdles that must be overcome before a truly competitive kesterite technology can be validated: [26]

- Necessary to reach $15 \%$ efficiency, on par with CdTe and CIGS technologies.

- Difficulty in growth of pure kesterite phase without secondary phases and stannite structure, which are particularly hard to be detected.

- Optimization of interfaces which is found to be important factor limiting device performance. As stated CdS interface with CZTS absorber layer shows excellent band offset, but the incorporation of $\mathrm{Cd}$ is not welcome everywhere. Because $\mathrm{Cd}$ is one of the six toxic material which hinders both environmental and human health acceptance. There are some other potential interfacial materials like $\mathrm{ZnS}, \mathrm{In}_{2} \mathrm{Sn}_{3}, \mathrm{ZnSe}, \mathrm{Zn}_{\mathrm{x}} \mathrm{Cd}_{1-\mathrm{x}} \mathrm{S}$ etc., which have their own merits and demerits and performance of the cell largely depends on this.

- The long term stability under heat, light, moisture should be confirmed and compared with its sister competitors like CdTe and CIGS.

- A truly low-cost and high throughput approach needs to be established, without sacrificing on performance, in order to meet a $<\$ 1 \mathrm{Wp}$ price target.

- Overall, the proper fabrication process of CZTS, composition of different elements, Grain boundary, surface quality controlling is yet to be explored. 


\section{Dye Sensitized Solar Cell}

Dye Sensitized Solar Cells (DSSCs) are placed in the category of third generation photovoltaics (PV) where new trends in the photovoltaic technology are applied. Among the different possibilities of $3^{\text {rd }}$ generation solar cells DSSC have the most promising prospect due to some attractive features, such as, simpler conventional roll-printing manufacturing techniques, semi-flexible and semi-transparent structure, which offers some versatile applicable areas to glass-based systems, especially in Building Integrated PV(BIPV), and most importantly, the materials used are low-cost. The overall efficiency of $\sim 12 \%$ (for laboratory small size) placed DSSCs as potential inexpensive alternatives to solid state devices. [27]

It is based on a semiconductor formed between a photo-sensitized anode and an electrolyte, a photo electro chemical system. A modern DSSC is composed of a porous layer of titanium dioxide nano particles, covered with a molecular dye that absorbs sunlight. The titanium dioxide which acts like an anode is immersed under an electrolyte solution, above which is a platinum-based catalyst which acts like a cathode.

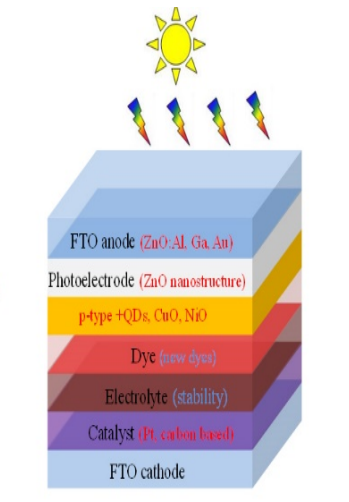

Structure of dye-sensitized solar cell.

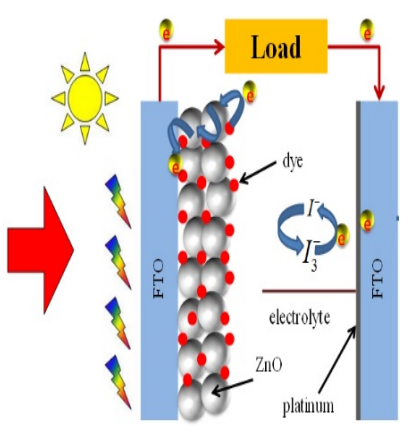

DDNO mechanism

Fig.7.DSSC sample structures and working mechanisms

Sunlight passes through the transparent electrode into the dye layer where it can excite electrons that then flow into the titanium dioxide. The electrons flow toward the transparent electrode where they are collected for powering a load. After flowing through the external circuit, they are re-introduced into the cell on a metal electrode on the back, flowing into the electrolyte. The electrolyte then transports the electrons back to the dye molecules.

There are lots of potential advantages of DSSC, which are -

- DSSCs are attractive as a replacement for existing technologies in "low density" applications like rooftop solar collectors, where the mechanical robustness and light weight of the glass-less collector is a major advantage.

- In quantum efficiency terms, DSSCs are extremely efficient. Due to their "depth" in the 
nanostructure there is a very high chance that a photon will be absorbed, and the dyes are very effective at converting them to electrons. The quantum efficiency of traditional designs varies, depending on their thickness, but are about the same as the DSSC.

- Differential kinetics of electron transfer from the Dye to $\mathrm{TiO}_{2}$ provides an extra advantage in case of DSSC. The rate of electron generation from the Dye is very fast and the recombination of the generated electron back to dye is rather very slow process. So as a consequence of this, DSSCs work even in low-light conditions. DSSCs are therefore able to work under cloudy skies and non-direct sunlight, whereas traditional designs would suffer a "cutout" at some lower limit of illumination, when charge carrier mobility is low and recombination becomes a major issue. The cutoff is so low they are even being proposed for indoor use, collecting energy for small devices from the lights in the house. [28]

- Temperature hardness is a very remarkable advantage of the DSSC, which means its performance is remarkably insensitive to temperature change. Thus, raising the temperature from 20 to $60{ }^{\circ} \mathrm{C}$ has no effect on the power conversion efficiency. Stability studies have shown the DSSC sustain temperatures of $85^{\circ} \mathrm{C}$ without loss of performance.

- A practical advantage, one DSSCs share with most thin-film technologies, is that the cell's mechanical robustness indirectly leads to higher efficiencies in higher temperatures. DSSCs are normally built with only a thin layer of conductive plastic on the front layer, allowing them to radiate away heat much easier, and therefore operate at lower internal temperatures.

Although the DSSC exhibits lots of promises, but there are still some drawbacks of such cells which requires more to explore -

- The major research issue of DSSC design is the use of the liquid electrolyte. This liquid has temperature stability problems. At low temperatures the electrolyte can freeze, ending power production and potentially leading to physical damage. Higher temperatures cause the liquid to expand, making sealing the panels a serious problem.

- In practice it has proven difficult to eliminate a number of expensive materials, notably platinum and ruthenium, and the liquid electrolyte presents a serious challenge to making a cell suitable for use in all weather.

- The dye molecules are quite small (nanometer sized), so in order to capture a reasonable amount of the incoming light the layer of dye molecules needs to be made fairly thick, much thicker than the molecules themselves. 
- The instability of the dye solar cell was identified as a main challenge. Its efficiency however, improved during the course of the time by optimizing the porosity, but the instability remained a problem. [29]

- A third major drawback is that the electrolyte solution contains volatile organic compounds (or VOC's), solvents which must be carefully sealed as they are hazardous to human health and the environment. This, along with the fact that the solvents permeate plastics, has precluded largescale outdoor application and integration into flexible structure. [30]

- Replacing the liquid electrolyte with a solid has been a major ongoing field of research. Recent experiments using solidified melted salts have shown some promise, but currently suffer from higher degradation during continued operation, and are not flexible. [31]

- Most of the small losses that do exist in DSSC's are due to conduction losses in the $\mathrm{TiO} 2$ and the clear electrode, or optical losses in the front electrode.

- For the future improvement of the conversion efficiency, understanding and control of interfacial effects are essential, as such type of cells have high contact area of the junction.

- It is important to determine the nature of the exposed surface planes of the oxide and the mode of interaction with the dye. [32]

- At this stage the confirmed efficiency obtained with the black dye is $10.4 \%$.[33]

Further development will concentrate on the enhancement of the photo response in the near IR region. The goal is to obtain a DYSC having optical features similar to GaAs. A nearly vertical rise of the photocurrent close to the $920 \mathrm{~nm}$ absorption threshold would increase the short circuit photocurrent from currently 20.5 to about $28 \mathrm{~mA} / \mathrm{cm} 2$. With the Voc and FF values would raise the overall efficiency to $14.2 \%$. [34]

\section{Conclusion}

A solar cell can be termed as "promising" if the cell can be manufactured by using the cheapest as well as most abundant materials without sacrificing energyproduction efficiency. All the solar cell design discussed here have the same goal. Perovskite cells have received tremendous attention in the public, as their research efficiencies recently soared above $20 \%$. They also offer a wide spectrum of low-cost applications. [35-37] Concerns with the price and availability of indium in CIGS and tellurium in CdTe, as well as toxicity of cadmium have been a large motivator to search for alternative thin film solar cell materials, CZTS as it is composed of earth abundant and non-toxic elements, it can outperform CIGS or CdTe based thin film cells. CZTS have increased 
efficiency to $12.0 \%$ in laboratory cells, but more work is needed for their commercialization. [38] Though the DSSC shows a lots of attractive features, it has proven difficult to eliminate a number of expensive materials, notably platinum and ruthenium, and the liquid electrolyte presents a serious challenge to making a cell suitable for use in all weather. Although its conversion efficiency is less than the best thin-film cells, in theory its price/performance ratio should be good enough to allow them to compete with fossil fuel electrical generation by achieving grid parity. [39]

\section{References}

[1] Shockley, W.; Queisser, H. J. (1961). "Detailed Balance Limit of Efficiency of p-n Junction Solar Cells". Journal of Applied Physics 32 (3): 510

[2] Green, M. A. (2001). "Third generation photovoltaics: Ultra-high conversion efficiency at low cost". Progress in Photovoltaics: Research and Applications 9 (2): 123. doi:10.1002/pip.360.

[3] Martí, A.; Luque, A. (1 September 2003). Next Generation Photovoltaics: High Efficiency through Full Spectrum Utilization. CRC Press. ISBN 978-1-4200-3386-1.

[4] Conibeer, G. (2007). "Third-generation photovoltaics". Materials Today 10 (11): 42. doi:10.1016/S1369-7021(07)70278-X
Is Perovskite the Future of Solar Cells? Engineering.com. December 6, 2013.

[5] Collavini, S., Völker, S. F. and Delgado, J. L. (2015). "Understanding the Outstanding Power Conversion Efficiency of Perovskite-Based Solar Cells". Angewandte Chemie International Edition 54 (34): 9757-9759.

[6] Kojima, Akihiro; Teshima, Kenjiro; Shirai, Yasuo; Miyasaka, Tsutomu (May 6, 2009). "Organometal Halide Perovskites as Visible-Light Sensitizers for Photovoltaic Cells". Journal of the American Chemical Society 131 (17): 6050-6051

\section{NREL Efficiency Chart}

[8] Eames, Christopher; Frost, Jarvist M.; Barnes, Piers R. F.; o'Regan, Brian C.; Walsh, Aron; Islam, M. Saiful (2015). "Ionic transport in hybrid lead iodide perovskite solar cells". Nature Communications 6: 7497

[9] Eperon, Giles E.; Stranks, Samuel D.; Menelaou, Christopher; Johnston, Michael B.; Herz, Laura M.; Snaith, Henry J. (2014). "Formamidinium lead trihalide: a broadly tunable perovskite for efficient planar heterojunction solar cells". Energy \& Environmental Science 7 (3): 982. oxford-pv-introduction-152.pdf 
[12] Eperon, Giles E.; Stranks, Samuel D.; Menelaou, Christopher; Johnston, Michael B.; Herz, Laura M.; Snaith, Henry J. (2014). "Formamidinium lead trihalide: a broadly tunable perovskite for efficient planar heterojunction solar cells". Energy \& Environmental Science 7 (3): 982

[13] Stranks, S. D.; Eperon, G. E.; Grancini, G.; Menelaou, C.; Alcocer, M. J. P.; Leijtens, T.; Herz, L. M.; Petrozza, A.; et al. (October 17, 2013). "Electron-Hole Diffusion Lengths Exceeding 1 Micrometer in an Organometal Trihalide

PerovskiteAbsorber". Science 342(6156): $\quad 341-$ 344

[14] Oxford Researchers Creating Simpler, Cheaper Solar Cells". SciTechDaily.com. November 12, 2013.

[15] D'Innocenzo, Valerio; Grancini, Giulia; Alcocer, Marcelo J. P.; Kandada, Ajay Ram Srimath; Stranks, Samuel D.; Lee, Michael M.; Lanzani, Guglielmo; Snaith, Henry J.; et al. (April 8, 2014). "Excitons versus free charges in organolead tri-halide perovskites". Nature Communications 5

[16] Habisreutinger, Severin N.; Leijtens, Tomas; Eperon, Giles E.; Stranks, Samuel D.; Nicholas, Robin J.; Snaith, Henry J. (2014). "Carbon
[17] M. LaMonica, "IBM Breaks Efficiency Mark with Novel Solar Material," Technology Review, 20 Aug 12.

[18] M. A. Green et al., "Solar Cell Efficiency Tables (Version 40)," Prog. Photovoltaic. Res. Appl. 20, 606 (2012).

[19] http://www.solar-facts-and advice.com/CZTS.html.

[20] S. Chen, X.G. Gong, A. Walsh, S.H. Wei, Appl. Phys. Lett. 96, 021902 (2010)

[21] K. Ito, T. Nakazawa, Jpn J Appl. Phys. 27, 2094 (1988)

[22] C.P. Chan, H. Lam, C. Surya, Solar Energy Mater. SolarCells 94, 207 (2010)

[23] W. Xinkun, L. Wei, C. Shuying, L. Yunfeng, J. Hongjie,J. Semiconductors 33, 022002 (2012)

[24] W. Shockley, H.J. Queisser, J. Appl. Phys. 32, $510(1961)$

[25] USGS, Commodity Statistics and Information, USGS Minerals Information (2010) 
[26] Yanqing Lai, et al,: Current status and future prospects of kesterite CZTS thin film solar cells.

[27] Yella A, Lee HW, Tsao HN, Yi C, Chandiran A.K, Nazeeruddin M.K, Diau EW, Yeh CY, Zakeeruddin SM, Grätzel. M. PorphyrinSensitized Solar Cells with Cobalt (II/III)-Based Redox Electrolyte Exceed 12 Percent Efficiency. Science 334, pp. 629-634, (2011)

[28] Kimberly Patch, "Solar cell doubles as battery", Technology Research News, 2006

[29] Matsumura, M. et all, "Dye Sensitization and Surface Structures of Semiconductor Electrodes". Ind. Eng. Chem. Prod. Res. Dev. 19 (3): 415421(1980).

[30] Ecole Polytechnique Fédérale de Lausanne, "New Efficiency Benchmark For Dye-sensitize solar Cells", ScienceDaily, 3 November 2008.

[31] Nathalie Rossier-Iten, "Solid hybrid dyesensitized solar cells: new organic materials, charge recombination and stability", École Polytechnique Fédérale de Lausanne, 2006. 